\title{
Selected topics on the interaction between cirrus clouds and embedded contrails
}

\author{
K. Gierens \\ Deutsches Zentrum für Luft- und Raumfahrt, Institut für Physik der Atmosphäre, Oberpfaffenhofen, Germany \\ Correspondence to: K. Gierens (klaus.gierens@dlr.de)
}

Received: 10 September 2012 - Published in Atmos. Chem. Phys. Discuss.: 24 September 2012

Revised: 28 November 2012 - Accepted: 12 December 2012 - Published: 17 December 2012

\begin{abstract}
Persistent contrails and natural cirrus clouds often coexist in the upper troposphere and contrails can be embedded within cirrus clouds. The present paper deals with some questions regarding the interaction of cirrus clouds and embedded contrails. I have selected only questions that can be answered by analytical means. I find that (1) the emission index for water vapour is only slightly changed when an aircraft crosses a cirrus cloud, (2) that contrail formation is not affected by an ambient cirrus, (3) that cirrus ice crystals entrained into the trailing wing tip vortex do not efficiently retard the sublimation of contrail ice crystals, and (4) that cirrus can start to dissolve an embedded contrail after a couple of hours by aggregation.
\end{abstract}

\section{Introduction}

Although contrails and cirrus clouds do not have the same formation conditions, they often occur together in the same regions because the condition for cirrus formation, ice supersaturation, is also required for contrail persistence. Indeed, sensitive observations with lidar instruments that are capable to detect optically very thin cirrus, including subvisible cirrus, suggest that contrails mostly occur together with cirrus (Sassen, 1997; Immler et al., 2008; Iwabuchi et al., 2012). Further, airborne lidar observations often show backscatter peaks within cirrus clouds that can be interpreted as embedded contrails.

A recent study of air traffic effects in the North Atlantic on cirrus (and contrail) coverage and outgoing longwave radiation (OLR, Schumann and Graf, 2012) showed an effect on OLR that was too large to be explainable by the additional cloud coverage due to contrails ( 1 to $2 \%$ ) alone. Instead, one must assume that aircraft flying through cirrus clouds produce contrails and further effects within these natural clouds which eventually manifests itself as a significant change in OLR. These findings are consistent with results from radiation transfer calculations (Schumann et al., 2012).

These results warrant to start studies concerning the interaction between cirrus clouds and embedded contrails using numerical models and analytical calculations where possible. To my knowledge, contrails have so far been modelled mainly as isolated objects and numerical simulations of the development of contrails within cirrus clouds have only recently been performed for the first time (Gierens and Spichtinger, in preparation). In the present paper I will present some simple analytical calculations on certain aspects of contrail development within cirrus clouds. Generally, the questions treated are of the kind "is process $\mathrm{P}$ important for the interaction or not?" and these questions can often be treated considering and comparing various time scales.

The questions treated are the following:

- How much do ice crystals sucked through an engine change the water vapour emission index?

- Is contrail formation within a cirrus affected by the cirrus?

- Can cirrus crystals mixed into the downward travelling wing vortex enhance the available water mass such that the sublimation of contrail ice is efficiently retarded?

- What is the role of aggregation between cirrus and contrail ice crystals?

These questions are dealt with in several sections. A summary is given at the end and since quite a number of 
calculations are performed I provide a nomenclature section as an appendix.

\section{How much do ice crystals sucked through an engine change the water vapour emission index?}

An aircraft flying through a cirrus cloud sucks ice crystals together with air through its engines. The ice sublimates and the resulting water vapour at engine exit adds to the water vapour resulting from burning kerosene; thus an enhanced emission index for water vapour results which could affect contrail formation. How large is this effect?

Let us assume a fairly large ice mixing ratio (water mass divided by air mass) of $q_{\mathrm{c}}=10^{-3.5}$. This value corresponds to the maximum mixing ratio measured in 28 research flights (see Schiller et al., 2008, their Fig. 2 and the second entry in their Table 2). An aircraft engine typically has an air fuel ratio $\dot{m}_{\text {air }} / \dot{m}_{\text {fuel }} \approx 70$, and the emission index of water vapour for kerosene is $\mathrm{EI}_{\mathrm{H}_{2} \mathrm{O}}=1.25$. With these values we find the ratio of water vapour emission at engine exit of sublimated ice vs. emission from kerosene combustion is $\left(\dot{m}_{\text {air }} q_{\mathrm{c}}\right) /\left(\dot{m}_{\text {fuel }} \mathrm{EI}_{\mathrm{H}_{2} \mathrm{O}}\right)$. This ratio is merely of the order percent even if the ice mixing ratio is very large.

It is instructive to compare this effect with the effect of varying water vapour emission indices. IPCC (1999, Sect. 7.8.2) gives a range of $1.25 \pm 0.03$ for $\mathrm{EI}_{\mathrm{H}_{2} \mathrm{O}}$, that is, a variation of $\pm 2.4 \%$ which compares to the effect of evaporating cirrus ice in very thick clouds. However, both these variations are probably dwarfed by the variation of $\mathrm{EI}_{\mathrm{H}_{2} \mathrm{O}}$ from alternative fuels. The NASA-AAFEX campaign (Anderson et al., 2011, table 2) gives a fuel H/C (hydrogen to carbon ratio) variation of more than $10 \%$ which might directly translate into a corresponding variation of $\mathrm{EI}_{\mathrm{H}_{2} \mathrm{O}}$. Compared to these variations, the contribution of evaporating ice from even the thickest cirrus clouds is negligible.

\section{Is contrail formation within a cirrus affected by the cirrus?}

How could a pre-existing cirrus affect contrail formation? When ambient air (i.e. cirrus air) is mixed into the exhaust plume, the plume temperature decreases and the relative humidity in the plume increases. Contrail formation requires that the relative humidity increases up to water saturation (Schmidt-Appleman criterion, see Schumann, 1996); if water saturation is reached, the water that is condensed on the exhaust particles, mainly on soot particles which serve as freezing nuclei, freezes and a contrail results. If this happens within a cirrus, ice crystals are mixed into the plume and start to grow as soon as the plume air is supersaturated with respect to ice, thereby consuming the excess vapour. Thus we have two competing processes affecting the plume supersaturation, the cooling due to mixing caused by jet dynamics and the growing ice crystals mixed into the plume from the am- bient cirrus. The latter process adds latent heat to the plume but this effect is negligible.

The temporal change of the saturation ratio $S(t)=$ $e(t) / e^{*}[T(t)]$ in an exhaust plume is given as

$$
\begin{aligned}
\frac{\mathrm{d} S}{\mathrm{~d} t} & =\frac{e^{*}[T(t)] \frac{\mathrm{d} e}{\mathrm{~d} t}-e(t) \frac{\mathrm{d} e^{*}}{\mathrm{~d} T} \frac{\mathrm{d} T}{\mathrm{~d} t}}{e^{*}[T(t)]^{2}} \\
& =\frac{1}{e^{*}[T(t)]} \frac{\mathrm{d} e}{\mathrm{~d} t}-S(t) \frac{L}{R_{\mathrm{V}} T^{2}} \frac{\mathrm{d} T}{\mathrm{~d} t} .
\end{aligned}
$$

The symbols are listed in the nomenclature section below. The second right hand side (rhs) term describes the change of the saturation vapour pressure due to jet cooling with a rate $\mathrm{d} T / \mathrm{d} t$ which I prescribe as a function $C(t)$ that is determined by the entrainment of ambient air into the hot plume air. Since the latent heat release is a negligible contribution to this term, it is not affected by the presence of an ambient cirrus and I do not consider it further.

The first rhs term describes change of $S$ due to the change of the vapour pressure $e$. This term has a jet dynamical and a microphysical component as follows:

$\frac{\mathrm{d} e}{\mathrm{~d} t}=G C(t)-c(t)$,

where $G$ is the change in vapour pressure per change in temperature of the isobarically expanding plume (cf. Schumann, 1996):

$G=\frac{c_{p} p \mathrm{EI}_{\mathrm{H}_{2} \mathrm{O}}}{\epsilon Q(1-\eta)}$.

The condensation function $c(t)$ for condensation on preexisting cirrus ice crystals can be approximated following Gierens (2003) as

$c(t)=\frac{e-e^{*}}{\tau_{\mathrm{g}}}$

with the crystal growth time scale $\tau_{\mathrm{g}}$ (see, e.g. Kärcher and Lohmann, 2002; Gierens, 2003). In order to see whether an ambient cirrus affects contrail formation it suffices to compare the two $S$-change rates $G C(t) / e^{*}$ and $c(t) / e^{*}$ or their inverses which can be interpreted as the respective time scales, that is $\tau_{\text {jet }}$ and $\tau_{\text {cond }}$, respectively. $C(t)$ can be obtained from an explicit consideration of the entrainment of ambient air into the jet where we have (Schumann, 1996)

$c_{p}\left(T_{\text {jet }}-T_{\text {cirrus }}\right) \approx \frac{Q(1-\eta)}{N(t)}$.

$N(t)$ is the plume dilution ratio, indicating that at plume age $t$ the jet is composed of one unit exhaust air and $N-1$ units of ambient cirrus air. In the equation above, I simply considered $c_{p}$ to be a constant and I neglected the enthalpy of ambient air against that of the hot exhaust gases which are reasonable approximations. $N(t)$ can be approximated from engine exit 
up to almost $3 \mathrm{~h}$ plume age as a power law (Schumann et al., 1998):

$N(t)=N_{0}\left(t / t_{0}\right)^{\gamma}$

where $N_{0}=7000$ when $t_{0}=1 \mathrm{~s} . \gamma=0.8$ was successfully used by Schumann et al. (1998) to fit a large number of insitu measurements. The cooling rate is then:

$C(t) \equiv \frac{\mathrm{d} T_{\mathrm{jet}}}{\mathrm{d} t}=-\frac{Q(1-\eta)}{c_{p}} \frac{\gamma}{N(t) t}$

Setting together equations 3 and 7 into the definition of $\tau_{\text {jet }}$, the dynamic time scale which characterises the mean dilution in the jet (of course the absolute value has to be taken), we find:

$\tau_{\text {jet }} \equiv \frac{e^{*}}{G C(t)}=\frac{\epsilon e^{*} N(t) t}{\mathrm{EI}_{\mathrm{H}_{2} \mathrm{O}} p \gamma}=0.62 \frac{e^{*}}{p} N(t) t$.

In order to find a typical order of magnitude for $\tau_{\text {jet }}$ we can take $t=1 \mathrm{~s}, N=N_{0}=7000$ and $e^{*} / p=\mathcal{O}\left(10^{-3}\right)$ which gives a timescale of a few seconds.

The condensation time scale can be obtained using expressions from Gierens (2003):

$\tau_{\mathrm{g}}^{-1}=1.4 \times 10^{-6} T^{1.61} p^{-1}\left(s e^{*}\right)^{1 / 3} \mathcal{N}_{\text {cir }}^{2 / 3}$,

which can be inserted into Eq. (4) and gives the condensation timescale as

$\tau_{\text {cond }}=\tau_{\mathrm{g}} / s$

with $s=S-1$.

Figure 1 shows the ratio $\tau_{\text {cond }} / \tau_{\text {jet }}$ as a function of cirrus ice crystal number concentration and plume age within the first second of the plume and for various ambient conditions. In order to yield a maximum effect of the ambient cirrus I have assumed water saturation for the value of $s$. In spite of this, the ratio is much larger than one for realistic cirrus conditions $\left(\mathcal{N}_{\text {cir }}<10 \mathrm{~cm}^{-3}\right)$. In microphysical terms: the cirrus crystals grow too slowly to efficiently reduce the increase of plume supersaturation due to the cooling. This means that contrail formation is not affected by the presence of an ambient cirrus cloud. A ratio of unity between the two time scales is only reached at very high crystal concentrations, $\mathcal{N}=\mathcal{O}\left(10^{4} \ldots 10^{5}\right) \mathrm{cm}^{-3}$. Such high concentrations are perhaps observed in the contrails themselves. Thus it is eventually the growing contrail ice that is able to compete with the dynamic jet cooling for affecting plume supersaturation. The number of particles that can freeze at sufficient supersaturations in a plume is much higher than the number of soot particles (which usually nucleate ice first). If there were not enough soot for letting $\tau_{\text {cond }} / \tau_{\text {jet }} \rightarrow 1$ other particles would freeze until eventually $\tau_{\text {cond }} / \tau_{\text {jet }}=1$ (see also Kärcher and Yu, 2009) .
4 Can cirrus crystals mixed into the downward travelling wing vortex enhance the available water mass such that the sublimation of contrail ice is efficiently retarded?

During the jet phase, cirrus ice crystals are entrained into the exhaust plume as enthalpy and water vapour are mixed in. Once the vortex pair is completely rolled up and starts to travel downwards, both contrail ice and cirrus ice crystals experience the adiabatic heating in the plume and start to sublimate as soon as the in-plume relative humidity decreases below ice saturation. Now, if only contrail ice is present, a certain fraction (depending mainly on initial supersaturation and temperature) of the contrail ice crystals eventually sublimates completely (Unterstrasser and Sölch, 2010). When cirrus ice is present, this process could be slowed down because the large cirrus crystals could supply water vapour without sublimating completely and the added water vapour helps to keep the relative humidity closer to ice saturation than without the cirrus crystals. In the following I estimate whether this can be an important process.

It suffices to compare the sublimation time scales for the two ice populations, the contrail ice and the cirrus ice. The depositional growth of a single ice crystal can conveniently be parameterised using the parameterisation by Koenig (1971), $d m / d t=a m^{b}$, where $m$ is the mass of a single crystal and where $a, b$ are parameters depending mainly on temperature and saturation ratio $(a<0$ for $S<1)$. Now, we have to generalise this formulation for a population of ice crystals with a certain crystal mass distribution and ice water concentration (IWC), $\mathcal{M}$. Integrating the single-crystal parameterisation over the crystal mass distribution results in the following formulation (Eq. (45) of Spichtinger and Gierens, 2009, without corrections for ventilation and kinetic effects):

$$
\frac{\mathrm{d} \mathcal{M}}{\mathrm{d} t}=a \mu_{b},
$$

where $\mu_{b}$ is the moment of order $b$ of the crystal's mass probability density function (pdf). $\mathcal{M}$ itself is proportional to the first moment, $\mathcal{M} \propto \mu_{1}$, and the sublimation timescale is then

$\tau=\frac{\mathcal{M}}{|\mathrm{d} \mathcal{M} / \mathrm{d} t|} \propto \frac{\mu_{1}}{\mu_{b}}$.

The moments $\mu_{k}$ are given in Spichtinger and Gierens (2009, Eq. 24) for a log-normal pdf, and these will be used here as well:

$\mu_{k}=\mathcal{N} \bar{m}^{k} r_{0}^{k(k-1) / 2}$,

with crystal number density $\mathcal{N}$ (the pdf is normalised to the number concentration of ice crystals), mean mass $\bar{m}$ and a parameter $r_{0}$ that quantifies the width of the pdf. With this information we can now compute the ratio of the sublimation 
time scales for contrail and cirrus ice:

$$
\begin{aligned}
\frac{\tau_{\mathrm{cir}}}{\tau_{\mathrm{con}}} & =\frac{\mathcal{N}_{\mathrm{cir}} \bar{m}_{\mathrm{cir}} r_{0, \mathrm{cir}}}{\mathcal{N}_{\mathrm{con}} \bar{m}_{\mathrm{con}} r_{0, \mathrm{con}}} \cdot \frac{\mathcal{N}_{\mathrm{con}} \bar{m}_{\mathrm{con}}^{b} r_{0, \mathrm{con}}^{b(b-1) / 2}}{\mathcal{N}_{\mathrm{cir}} \bar{m}_{\mathrm{cir}}^{b} r_{0, \mathrm{cir}}^{b(b-1) / 2}} \\
& =\frac{\bar{m}_{\mathrm{cir}}^{1-b} r_{0, \mathrm{cir}}^{1-b(b-1) / 2}}{\bar{m}_{\mathrm{con}}^{1-b} r_{0, \mathrm{con}}^{1-b(b-1) / 2}} .
\end{aligned}
$$

The width parameters $r_{0}$ of cirrus and contrail pdfs are similar (values of 2 to 4 are typical) such that their ratio is of the order unity. The value of $b$ is of the order $1 / 3$ to $1 / 2$, such that also the exponent $1-b(b-1) / 2$ approximates unity; the factors involving the width parameters can thus be safely neglected. What remains is the ratio of the mean crystal masses, viz.:

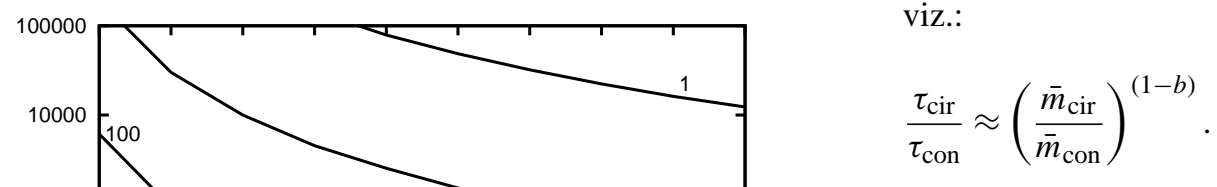

This ratio is typically of the order 100 . Thus the effect of cirrus ice in the downward travelling plume to halt the sublimation of the contrail ice is usually small or even negligible. This result is surprising if we compare the total ice mass concentrations in the plume: $\mathcal{M}_{\text {con }}$ is typically of the order 1 to $10 \mathrm{mg} \mathrm{m}^{-3}$ (Schumann, 2002, Fig. 11.5) while that of a cirrus can reach a few $10 \mathrm{mg} \mathrm{m}^{-3}$ (e.g. Schiller et al., 2008). Although there can be more cirrus ice in the contrail vortex than contrail ice itself, the reaction of the cirrus ice to changing saturation conditions is much too slow to retard the sublimation of the contrail ice when subsaturation is reached in the adiabatically heated plume.

\section{What is the role of aggregation between cirrus and contrail ice crystals?}

After the end of the vortex phase the contrail develops further within the cirrus cloud. There is generally a complex interaction between the two ice classes, depending on ambient conditions, on the state of the cirrus, on the composition of the cirrus ice crystals (heterogeneously vs. homogeneously formed ice crystals) and so on. Thus numerical simulations are generally required to capture all these possible interactions. But one can at least estimate the role of aggregation between cirrus and contrail ice crystals. In particular if the contrail is located in the lower part of a cirrus layer it is possible that large cirrus crystals falling from above into the contrail collect contrail ice crystals, thereby diminishing the contrail signature in the perturbed cloud.

I derive the expression for the aggregation time scale starting from the master equation for aggregation (cf. KienastSjögren et al., 2012). Assuming that contrail crystals are depleted by aggregation with cirrus crystals and neglecting aggregation between contrail crystals, the master equation takes 
the simple form:

$\frac{\partial n\left(m_{\mathrm{con}}, t\right)}{\partial t}=-\int_{0}^{\infty} K\left(m_{\mathrm{con}}, m_{\mathrm{cir}}\right) n\left(m_{\mathrm{con}}, t\right) n\left(m_{\mathrm{cir}}, t\right) \mathrm{d} m_{\mathrm{cir}}$.

Here, $n(m, t)$ is a number density per infinitesimal mass interval. Using the normalized pdf $f(m, t)=n(m, t) / \mathcal{N}$ and integrating both sides over all contrail crystal masses we obtain:

$\frac{\partial \mathcal{N}_{\text {con }}(t)}{\partial t}=-\mathcal{N}_{\text {con }} \mathcal{N}_{\text {cir }} \times$

$\iint_{0}^{\infty} K\left(m_{\mathrm{con}}, m_{\mathrm{cir}}\right) f\left(m_{\mathrm{con}}, t\right) f\left(m_{\mathrm{cir}}, t\right) \mathrm{d} m_{\mathrm{cir}} \mathrm{d} m_{\mathrm{con}}$.

The double integral can be conceived as the expectation value of $K$ given the mass pdfs, $\langle K\rangle$. The aggregation time scale is then

$\tau_{\text {aggr }}=\frac{\mathcal{N}_{\text {con }}}{\left|\partial \mathcal{N}_{\text {con }} / \partial t\right|}=\left(\mathcal{N}_{\text {cir }}\langle K\rangle\right)^{-1}$,

For the aggregation kernel I assume the following expression (Field and Heymsfield, 2003; Westbrook et al., 2004):

$K=\pi\left(R_{\text {con }}^{2}+R_{\text {cir }}^{2}\right) v_{\mathrm{t}} E_{\mathrm{a}}$,

where $v_{\mathrm{t}}$ is the fall speed of the cirrus crystals. The fall speed of the contrail crystals has been neglected and that is the reason why I could neglect aggregation between contrail ice crystals in the master equation. This assumption is of course not generally valid, but for contrail crystals embedded in a near-saturated environment of a cirrus cloud I expect only weak growth and thus small fall speeds. $E_{\mathrm{a}}$ is the collision efficiency. As generally $R_{\text {con }}<R_{\text {cir }}$ we may simplify the analysis by also neglecting $R_{\text {con }}^{2}$ in the expression for $K$. The corresponding integral for $\langle K\rangle$ then reduces to a single integral over the cirrus crystal masses. The terminal velocity is usually taken proportional to $\left.R_{\text {cir }}^{y}: v_{\mathrm{t}}=v_{0}\left(R_{\mathrm{cir}} / R_{0}\right)^{y}\right)$. Using this we have

$\tau_{\text {aggr }}=\left(\mathcal{N}_{\text {cir }}\left\langle R_{\text {cir }}^{2+y}\right\rangle v_{0} \pi E_{\mathrm{a}}\right)^{-1}$.

$y$ is in the range 0.7 to 1.3 depending on crystal size and habit (e.g. Heymsfield and Iaquinta, 2000, Eq. A29), but for crystals with maximum dimension smaller than about $0.5 \mathrm{~mm}$ (which are much more abundant than larger ones), $y$ is about 1.25 , and the prefactor $v_{0}$ is of the order $22700 \mathrm{~m} \mathrm{~s}^{-1}$ for a unit radius $R_{0}=1 \mathrm{~m}$. With this parameterisation, an ice crystal of $R=100 \mu \mathrm{m}$ would fall with a speed of $23 \mathrm{~cm} \mathrm{~s}^{-1}$. For a lognormal radius pdf we can estimate $\left\langle R_{\text {cir }}^{3.25}\right\rangle \approx 2 \bar{R}^{3.25}$. With $E_{\mathrm{a}} \approx 0.7$ we can write the following approximation:

$\tau_{\text {aggr }} \approx 4 \times 10^{-5} \mathcal{N}_{\text {cir }}^{-1} \bar{R}_{\text {cir }}^{-3.25}$.

As shown in Fig. 2, this aggregation time scale varies between approximately $1 \mathrm{~s}$ for an unrealistically high density

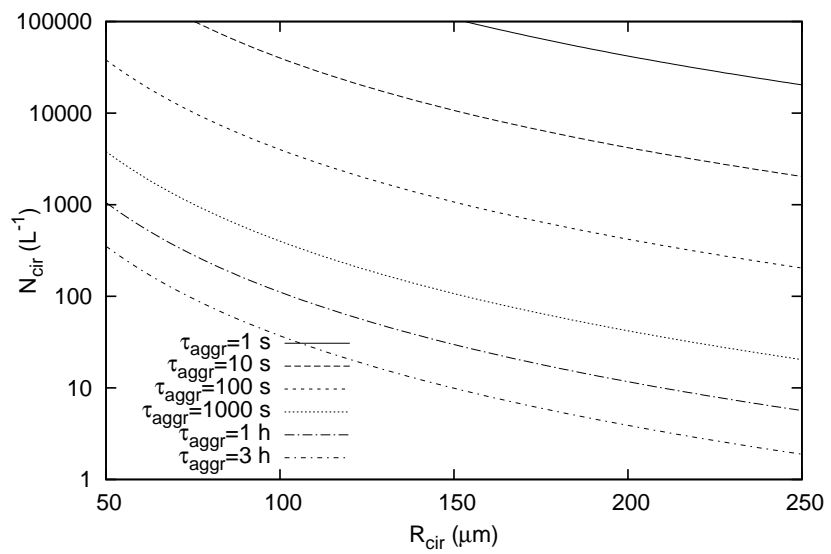

Fig. 2. Aggregation time scale for the removal of contrail ice crystals by sedimenting cirrus ice crystals as a function of the mean cirrus crystal radius $\bar{R}_{\text {cir }}$ and the cirrus crystal number concentration $\mathcal{N}_{\text {cir }}$. Note that only parameter combinations below the the main diagonal can be considered realistic in cirrus clouds; thus aggregation timescales are typically of the order hours.

of large crystals (the upper right corner in the figure) and several hours. Heymsfield and McFarquhar (2002, Fig. 4.6) present concentrations of large crystals with $R_{\text {cir }}>50 \mu \mathrm{m}$. These have typically concentrations of 1 to $101^{-1}$. Thus, aggregation timescales are typically a couple of hours, similar to timescales needed for contrails to spatially expand several kilometres. Thus we can expect that cirrus clouds after some hours start to remove the ice of embedded contrails (in particular contrails at cloud bottom) via aggregation.

\section{Summary}

As a prooemium to more elaborate numerical studies (Gierens and Spichtinger, in preparation), I have treated several questions regarding the interaction between cirrus and embedded contrails. Only questions that can be answered by analytical means, mainly by comparison of certain timescales, have been selected. The questions and their answers are:

Q: How much do ice crystals sucked through an engine change the water vapour emission index?

A: The emission index of water vapour changes by a few percent at most when an aircraft crosses a cirrus cloud.

Q: Is contrail formation within a cirrus affected by the cirrus?

A: No. The contrail forms as if no cirrus were present.

Q: Can cirrus crystals mixed into the downward travelling wing vortex enhance the available water mass such that the sublimation of contrail ice is efficiently retarded? 
A: Hardly, although the ice mass provided by the cirrus can be larger than the ice mass of the contrail itself. As the concentration of contrail crystals is several orders of magnitude larger than the concentration of cirrus crystals entrained in the plume, the cirrus crystals react slowly on changing saturation conditions and therefore have hardly any effect on the evolution of the saturation ratio within the plume, which is driven mainly by the vortex dynamics.

Q: What is the role of aggregation between cirrus and contrail ice crystals?

A: After a few hours it is possible that sedimenting cirrus crystals collect a significant fraction of contrail crystals (mainly for contrails embedded in the lower part of a cirrus), and thus that the contrail dissolution is enhanced by aggregation with cirrus crystals.

These answers can be used to justify certain simplifications in numerical models. For instance, contrail formation can be numerically treated in the same way whether the contrail is in clear air or embedded in a cirrus cloud since pre-existing ice does neither affect the water vapour emission index nor contrail formation itself in a significant way. Thus it is possible to follow a usual procedure in contrail modelling and start a simulation at the beginning of the vortex phase without explicitely modeling contrail formation in the early jet phase. Similarly, determination of initial conditions for dispersion phase simulations typically require the simulation of the vortex phase. The result of our third question shows that the presence of cirrus crystals within the vortex might be neglected, such that results of vortex phase simulations of contrails in clear air can be used to initialise dispersion phase simulations of embedded contrails.

There are many more interesting questions regarding contrails embedded in cirrus clouds. To answer them is generally beyond the possibilities of analytical estimation. Numerical simulations are then required which are the topic of another paper.

\section{Nomenclature}

$a$ prefactor for crystal growth parameterisation

$b \quad$ exponent for crystal growth parameterisation

c condensation rate in terms of partial pressure $\left(\mathrm{Pas}^{-1}\right)$

$c_{p} \quad$ specific heat of air $\left(1005.7 \mathrm{~J} \mathrm{~kg}^{-1} \mathrm{~K}^{-1}\right)$

$C \quad$ cooling rate due to jet expansion $\left(\mathrm{K} \mathrm{s}^{-1}\right)$

$e \quad$ partial pressure of water vapour $(\mathrm{Pa})$

$e^{*} \quad$ saturation (with respect to ice) partial pressure of water vapour $(\mathrm{Pa})$

$E_{\mathrm{a}} \quad$ collision and collection efficiency $(\approx 0.7)$

$\mathrm{EI}_{\mathrm{H}_{2} \mathrm{O}}$ emission index of water vapour (1.25) $f \quad$ normalised probability density function for crystal masses $\left(\mathrm{kg}^{-1}\right)$

$G \quad \mathrm{~d} e / \mathrm{d} T$ in an expanding plume $\left(\mathrm{PaK}^{-1}\right)$

$K \quad$ aggregation kernel $\left(\mathrm{m}^{3} \mathrm{~s}^{-1}\right)$

$L \quad$ latent heat of sublimation $\left(2.8352 \times 10^{6} \mathrm{~J} \mathrm{~kg}^{-1}\right)$

$\mathcal{M}$ ice water concentration (IWC, $\mathrm{kg} \mathrm{m}^{-3}$ )

$\dot{m}_{\text {air/fuel }}$ air and fuel flow rates through an engine $\left(\mathrm{kg} \mathrm{s}^{-1}\right)$

$m \quad$ crystal mass $(\mathrm{kg})$

$\bar{m} \quad$ mean mass of an ice crystal pdf $(\mathrm{kg})$

$n \quad$ number density of ice crystals per

infinitesimal mass interval $\left(\mathrm{m}^{-3} \mathrm{~kg}^{-1}\right)$

$\mathcal{N} \quad$ number density of ice crystals $\left(\mathrm{m}^{-3}\right)$

$N, N_{0} \quad$ plume dilution ratio, at unit time

$p \quad$ pressure $(\mathrm{Pa})$

$Q \quad$ calorific value of kerosene $\left(43.2 \mathrm{MJ} \mathrm{kg}^{-1}\right)$

$q_{\mathrm{c}} \quad$ ice mixing ratio $\left(\mathrm{kg}\right.$ ice $\left.(\mathrm{kg} \text { air })^{-1}\right)$

$r_{0} \quad$ width parameter of crystal size distribution

$R \quad$ crystal "radius" (m)

$\bar{R} \quad$ mean crystal radius (m)

$R_{\mathrm{V}} \quad$ gas constant of water vapour $\left(461.5 \mathrm{~J} \mathrm{~kg}^{-1} \mathrm{~K}^{-1}\right)$

$s \quad$ supersaturation with respect to ice $(S-1)$

$S \quad$ ice saturation ratio

$t \quad$ time or plume age (s)

$T, T_{x} \quad$ various temperatures $\left(\mathrm{K}\right.$ or $\left.{ }^{\circ} \mathrm{C}\right)$

$v_{\mathrm{t}} \quad$ terminal fall speed of ice crystals $\left(\mathrm{m} \mathrm{s}^{-1}\right)$

$y \quad$ exponent in fall speed to radius relation

$\gamma \quad$ exponent for plume dilution parameterization

$\epsilon \quad$ ratio of molar masses of water and air $(0.622)$

$\eta \quad$ overall propulsion efficiency of an aircraft $(\approx 0.3)$

$\tau_{x} \quad$ various process time scales (s)

Acknowledgements. I thank Ulrich Schumann for critically reading a preliminary version of this manuscript and Rick Miake-Lye and two anonymous reviewers for for their constructive comments. The work contributes to the DLR program "Emissionen und Klimawirkung des Luftverkehrs".

The service charges for this open access publication have been covered by a Research Centre of the Helmholtz Association.

Edited by: M. Krämer

\section{References}

Anderson, B. E., Beyersdorf, A. J., Hudgins, C.H., Plant, J. V., Thornhill, K. L., Winstead, E. L., Ziemba, L.D., Howard, R., Corporan, E., Miake-Lye, R. C., Herndon, S.C., Timko, M., Woods, E., Dodds, W., Lee, B., Santoni, G., Whitefield, P., Hagen, D., Lobo, P., Knighton, W. B., Bulzan, D., Tacina, K., Wey, C., Vander Wal, R., Bhargava, A., Kinsey, J., and Liscinsky, D. S.: Alternative Aviation Fuel Experiment (AAFEX), NASA/TM 2011-217059, 408 pp., 2011. 
Field, P. and Heymsfield, A.: Aggregation and scaling of ice crystal size distributions, J. Atmos. Sci., 56, 544-560, 2003.

Gierens, K.: On the transition between heterogeneous and homogeneous freezing, Atmos. Chem. Phys., 3, 437-446, doi:10.5194/acp-3-437-2003, 2003.

Heymsfield, A. and Iaquinta, J.: Cirrus crystal terminal velocities, J. Atmos. Sci., 57, 916-938, 2000.

Heymsfield, A. and McFarquhar, G.: Mid-latitude and tropical cirrus: microphysical properties, in: Cirrus, edited by: Lynch, D., Sassen, K., Starr, D., and Stephens, G., Oxford University Press, New York, USA, 78-101, 2002.

Immler, F., Treffeisen, R., Engelbart, D., Krüger, K., and Schrems, O.: Cirrus, contrails, and ice supersaturated regions in high pressure systems at northern mid latitudes, Atmos. Chem. Phys., 8, 1689-1699, doi:10.5194/acp-8-1689-2008, 2008.

Lewis, J.S., Niedzwiecki, R.W., and 30 coauthors: Aircraft technology and its relation to emissions. In: Aviation and the global atmosphere, J.E. Penner, D.H. Lister, D.J. Griggs, D.J. Dokken, and M. McFarland (eds.), IPCC Special Report, Cambridge University Press, UK, 373 pp., 1999.

Iwabuchi, H., Yang, P., Liou, K., and Minnis, P.: Physical and optical properties of persistent contrails: climatology and interpretation, J. Geophys. Res., 117, D06215, doi:10.1029/2011JD017020, 2012.

Kärcher, B. and Lohmann, U.: A parameterization of cirrus cloud formation: homogeneous freezing of supercooled aerosols, J. Geophys. Res., 107, 4698, doi:10.1029/2001JD000470, doi:10.1029/2001JD000470, 2002.

Kärcher, B. and Yu, F.: Role of aircraft soot emissions in contrail formation, Geophys. Res. Lett., 36, L01804, doi:10.1029/2008GL036649, 2009.

Kienast-Sjögren, E., Spichtinger, P., and Gierens, K.: Formulation and test of an ice aggregation scheme for two-moment bulk microphysics schemes, Atmos. Chem. Phys. Discuss., 12, 2397524009, doi:10.5194/acpd-12-23975-2012, 2012.
Koenig, L.: Numerical modeling of ice deposition, J. Atmos. Sci., 28, 226-237, 1971.

Sassen, K.: Contrail-cirrus and their potential for regional climate change, B. Am. Meteorol. Soc., 78, 1885-1903, 1997.

Schiller, C., Krämer, M., Afchine, A., Spelten, N., and Sitnikov, N.: Ice water content of Arctic, midlatitude, and tropical cirrus, J. Geophys. Res., 113, D24208, doi:10.1029/2008JD010342, 2008.

Schumann, U.: On conditions for contrail formation from aircraft exhausts, Meteorol. Z., 5, 4-23, 1996.

Schumann, U.: Contrail cirrus, in: Cirrus, edited by: Lynch, D., Sassen, K., Starr, D., and Stephens, G., Oxford University Press, chapter 11, 231-255, Oxford, New York, 2002.

Schumann, U. and Graf, K.: Aviation-induced cirrus and radiation changes at diurnal timescales, J. Geophys. Res., submitted, 2012.

Schumann, U., Schlager, H., Arnold, F., Baumann, R., Haschberger, P., and Klemm, O.: Dilution of aircraft exhaust plumes at cruise altitudes, Atmos. Environ., 32, 3097-3103, 1998.

Schumann, U., Mayer, B., Graf, K., and Mannstein, H.: A parametric radiative forcing model for contrail cirrus, J. Appl. Meteorol. Climatol., 51, 1391-1406, 2012.

Spichtinger, P. and Gierens, K. M.: Modelling of cirrus clouds Part 1a: Model description and validation, Atmos. Chem. Phys., 9, 685-706, doi:10.5194/acp-9-685-2009, 2009.

Unterstrasser, S. and Sölch, I.: Study of contrail microphysics in the vortex phase with a Lagrangian particle tracking model, Atmos. Chem. Phys., 10, 10003-10015, doi:10.5194/acp-1010003-2010, 2010.

Westbrook, C., Ball, R., Field, P., and Heymsfield, A.: Theory of growth by differential sedimentation, with application to snowflake formation, Phys. Rev. E, 70, 021403, 1-7, 2004. 